\title{
Image Forgery Detection Based On Sets of 9 Dimensional Convex Equations
}

\author{
Vinoth S \\ ECE Department \\ IIIT Hyderabad \\ India
}

Abstract: Image forgery creation is analyzed from 9 dimensional convex equation analysis

Keywords: 9 dimensions, convex equation

\section{INTRODUCTION}

Image forgery is created based on 9 dimensional convex equations and their analysis.

\section{EQUATIONS AND THEIR}

\section{ANALYSIS}

A 18 dimensional plane is considered. Bases are taken for each of the dimensions as $\left[\begin{array}{lllllll}1 & 0 & 0 & 0 & \ldots & 0\end{array}\right],\left[\begin{array}{lllllll}0 & 1 & 0 & 0 & 0 & \ldots & 0\end{array}\right],\left[\begin{array}{llllll}0 & 0 & 1 & 0 & 0\end{array}\right.$ $\left.\begin{array}{lll}0 & \ldots & 0\end{array}\right] \ldots\left[\begin{array}{lllll}0 & 0 & 0 & \ldots & 1\end{array}\right]$. Equations are considered by clubbing two bases in terms of a parabola with the ends symmetric to origin. Likewise 9 such combinations are considered to acheive 9 such convex shapes centered at origin.

Equation of 1st convex shape

$\mathrm{Y} 1=\mathrm{a} 1\left[\begin{array}{llllll}1 & 0 & 0 & 0 & \ldots & 0\end{array}\right]+\mathrm{b} 1\left[\begin{array}{llllll}0 & 1 & 0 & 0 & 0 & \ldots\end{array}\right]$. where $\mathrm{a} 1$ and $\mathrm{b} 1$ are vectors.

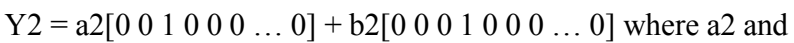
b2 are vectors.

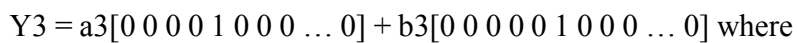
$\mathrm{a} 3$ and $\mathrm{b} 3$ are vectors.

$\mathrm{Y} 9=\mathrm{a} 9\left[\begin{array}{llllll}0 & 0 & 0 & \ldots & 1 & 0\end{array}\right]+\mathrm{b} 9\left[\begin{array}{llllll}0 & 0 & 0 & \ldots & 0 & 1\end{array}\right]$ where $\mathrm{a} 9$ and $\mathrm{b} 9$ are vectors.

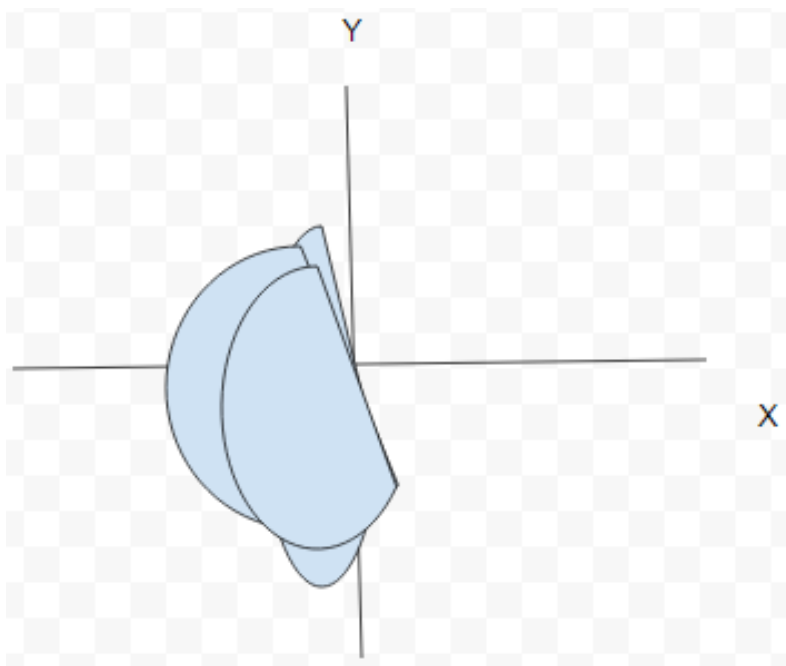

Fig. 1 Y1, Y2 and Y3

\section{CREATION OF FORGED IMAGE}

\subsection{Projection of considered image}

The image equation is calculated based on backpropagation neural network's weight vectors as seen in reference [2].

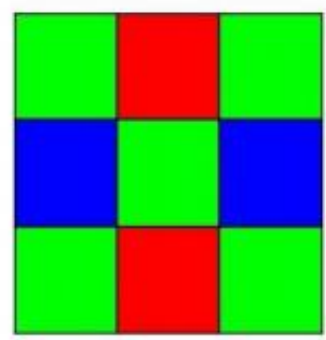

\begin{tabular}{|c|c|c|}
\hline G1 & g6 & G2 \\
\hline g7 & G5 & g9 \\
\hline G3 & g8 & G4 \\
\hline
\end{tabular}

Fig. 2 Calculation of image equation(a)

From Fig 2 [G1 G2 G3 G4 G5] are given as input and [g6 g7 g8 g9] are given as output in Fig 3. 

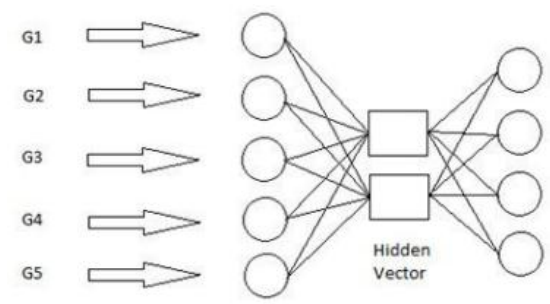

Input vector

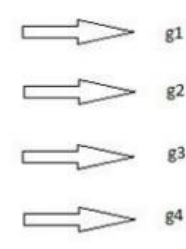

Output vector

Fig. 3 Calculation of image equation(b)

The neural networks are trained as seen above and the average of all weights stacked together forms the image equation. Average of all neural network weights is taken as the equation of the image considered. This equation is projected on the 9 different convex shapes considered above i.e. Y1 Y2 Y3 ... Y9.

\subsection{Detection of forged image}

Equations whose null spaces of projections on the 9 convex shapes are taken. If there is an intersection of this equation with that of the image equation the image is not forged.

Equations whose null spaces of projections on the 9 convex

shapes are taken. If there is an intersection of this equation with that of the image equation the image is not forged. This analysis is repeated for all the null spaces arrived for at the convex equational base. This is extended to one more dimension to seek whether the image is forged.

Projections are taken from this equation onto the convex shapes on these convex equations to create a forged image.

\section{ACKNOWLEDGMENTS}

Our thanks to the experts who have contributed towards development of the template.

\section{REFERENCES}

[1] Vinoth S, Image forgery analysis based on 9 dimensional convex equations

[2] Vinoth S, ES Gopi, Neural Network modelling of color array filter for image forgery detection using kernel LDA

[3] Vinoth S, Image forgery detection based on shape of eye ball

[4] Vinoth S, Image forgery detection based on 9 dimensional convex equations 